\title{
Special issue on multiagent interaction without prior coordination: guest editorial
}

\author{
Stefano V. Albrecht ${ }^{1}$. Somchaya Liemhetcharat ${ }^{2}$. \\ Peter Stone $^{1}$
}

Published online: 27 December 2016

(C) The Author(s) 2016

\begin{abstract}
This special issue of the Journal of Autonomous Agents and Multi-Agent Systems sought research articles on the emerging topic of multiagent interaction without prior coordination. Topics of interest included empirical and theoretical investigations of issues arising from assumptions of prior coordination, as well as solutions in the form of novel models and algorithms for effective multiagent interaction without prior coordination.

Interaction between agents is the defining attribute of multiagent systems, encompassing problems of planning in a decentralised setting, learning models of other agents, composing teams with high task performance, and selected resource-bounded communication and coordination. There is significant variety in methodologies used to solve such problems, including symbolic reasoning about negotiation and argumentation, distributed optimisation methods, machine learning methods such as multiagent reinforcement learning, etc. The majority of these well-studied methods depend on some form of prior coordination between agents. Often, the coordination is at the level of problem definition. For example, learning algorithms may assume that all agents share a common learning method or prior beliefs, distributed optimisation methods may assume specific structural constraints regarding the partition of state space and cost/rewards, and symbolic methods often make strong assumptions regarding norms and protocols. While such assumptions can facilitate effective interaction if all agents adhere to them, there are important applications in which prior coordination of agents may not be feasible or desirable. In particular, as a growing number of autonomous agents are being developed by different organisations and deployed in a variety of applications, it will be increasingly important for such agents to be able to interact effectively with other agents in the absence of prior agreements regarding action coordination and information sharing. Similar issues are also becoming increasingly pertinent in human-machine interactions, where there is a need for intelligent adaptive behaviour and assumptions regarding prior knowledge
\end{abstract}

Stefano V. Albrecht

svalb@cs.utexas.edu

1 The University of Texas at Austin, Austin, TX, USA

2 A*STAR, Singapore, Singapore 
and communication can be problematic. Thus, there has been a recent surge of interest on models and algorithms for multiagent interaction without prior coordination.

This special issue sought mature high-quality research related to multiagent interaction without prior coordination. Topics of interest included empirical and theoretical investigations of issues arising from assumptions of prior coordination, as well as solutions in the form of novel models and algorithms for effective multiagent interaction without prior coordination.

The submission deadline for manuscripts was December 18th, 2015. After an initial screening of submissions, nine submitted manuscripts were put forward for review. Each manuscript was reviewed by three selected experts in the respective area, based on relevance, novelty, significance, technical quality, and clarity. Two of the submitted manuscripts were (co-)authored by a guest editor of this special issue, and these were reviewed outside the editorial system and with no involvement of the respective guest editor. Following two rounds of reviews, five manuscripts were accepted for publication in this special issue.

The following articles appear in this special issue:

[1] Pablo Hernandez-Leal, Yusen Zhan, Matthew E. Taylor, L. Enrique Sucar, Enrique Munoz de Cote

Efficiently detecting switches against non-stationary opponents

[2] Katie Genter, Tim Laue, Peter Stone

Three Years of the RoboCup Standard Platform League Drop-In Player Competition: Creating and Maintaining a Large Scale Ad Hoc Teamwork Robotics Competition

[3] Muthukumaran Chandrasekaran, Prashant Doshi, Yifeng Zeng, Yingke Chen

Can Bounded and Self-Interested Agents be Teammates? Application to Planning in Ad Hoc Teams

[4] Alessandro Panella, Piotr Gmytrasiewicz

Interactive POMDPs with Finite-State Models of Other Agents

[5] Somchaya Liemhetcharat, Manuela Veloso

Allocating Training Instances to Learning Agents for Team Formation

These articles cover a range of important topics related to multiagent interaction without prior coordination. The article by Hernandez-Leal et al. [1] considers a multiagent setting in which unknown opponents can switch between multiple stationary strategies, and proposes a learning method which is able to detect and respond to switches in strategies. Genter et al. [2] provide a detailed description of the first three "Drop-in Player" competitions at RoboCup, in which robot players are drawn from different teams in the standard platform league and there is no prior coordination between players. Chandrasekaran et al. [3] consider agents engaged in a form of finite recursive reasoning (i.e. beliefs about beliefs) and show that this can lead to suboptimal team performance with self-interested agents, and how this can be alleviated using learning. Panella and Gmytrasiewicz [4] propose a variant of the Interactive POMDP model in which an autonomous agent learns finite-state controller models of other agents in partially observable domains, using a Bayesian nonparametric prior over the model parameters. Finally, Liemhetcharat and Veloso [5] consider a team formation problem in which agents have specific but unknown learning capabilities, and propose algorithms for the automatic allocation of training instances with the goal of finding an optimal team for a given task. Together, we believe these articles make significant contributions toward progress in multiagent interaction without prior coordination.

The guest editors would like to thank the reviewers for providing thoughtful and detailed manuscript reviews, as well as the editorial office staff at Springer for their assistance during the review process. 